\title{
BMJ Open Health economic burden that wounds impose on the National Health Service in the UK
}

\author{
Julian F Guest, ${ }^{1,2}$ Nadia Ayoub, ${ }^{1}$ Tracey Mcllwraith, ${ }^{1}$ Ijeoma Uchegbu, ${ }^{1}$ \\ Alyson Gerrish, ${ }^{1}$ Diana Weidlich, ${ }^{1}$ Kathryn Vowden, ${ }^{3}$ Peter Vowden ${ }^{3}$
}

To cite: Guest JF, Ayoub N, Mcllwraith T, et al. Health economic burden that wounds impose on the National Health Service in the UK. BMJ Open 2015;5: e009283. doi:10.1136/ bmjopen-2015-009283

- Prepublication history for this paper is available online. To view these files please visit the journal online (http://dx.doi.org/10.1136/ bmjopen-2015-009283).

Received 1 July 2015 Revised 19 October 2015 Accepted 20 October 2015

${ }^{1}$ Catalyst Health Economics Consultants, Northwood, Middlesex, UK

${ }^{2}$ Faculty of Life Sciences and Medicine, King's College, London, UK

${ }^{3}$ Bradford Teaching Hospitals NHS Foundation Trust and University of Bradford, Bradford, UK

\section{Correspondence to} Professor Julian F Guest; julian.guest@catalyst-health. com

\section{ABSTRACT}

Objective: To estimate the prevalence of wounds managed by the UK's National Health Service (NHS) in 2012/2013 and the annual levels of healthcare resource use attributable to their management and corresponding costs.

Methods: This was a retrospective cohort analysis of the records of patients in The Health Improvement Network (THIN) Database. Records of 1000 adult patients who had a wound in 2012/2013 (cases) were randomly selected and matched with 1000 patients with no history of a wound (controls). Patients' characteristics, wound-related health outcomes and all healthcare resource use were quantified and the total NHS cost of patient management was estimated at 2013/2014 prices.

Results: Patients' mean age was 69.0 years and $45 \%$ were male. $76 \%$ of patients presented with a new wound in the study year and $61 \%$ of wounds healed during the study year. Nutritional deficiency (OR 0.53; $p<0.001)$ and diabetes (OR $0.65 ; p<0.001$ ) were independent risk factors for non-healing. There were an estimated 2.2 million wounds managed by the NHS in $2012 / 2013$. Annual levels of resource use attributable to managing these wounds and associated comorbidities included 18.6 million practice nurse visits, 10.9 million community nurse visits, 7.7 million GP visits and 3.4 million hospital outpatient visits. The annual NHS cost of managing these wounds and associated comorbidities was $£ 5.3$ billion. This was reduced to between $£ 5.1$ and $£ 4.5$ billion after adjusting for comorbidities.

Conclusions: Real world evidence highlights wound management is predominantly a nurse-led discipline. Approximately $30 \%$ of wounds lacked a differential diagnosis, indicative of practical difficulties experienced by non-specialist clinicians. Wounds impose a substantial health economic burden on the UK's NHS, comparable to that of managing obesity ( $£ 5.0$ billion). Clinical and economic benefits could accrue from improved systems of care and an increased awareness of the impact that wounds impose on patients and the NHS.

\section{INTRODUCTION}

Patients requiring wound care can be found in the community, secondary care and in long-term care institutions and range from

\section{Strengths and limitations of this study}

- This study estimated the health outcomes, resource implications and associated costs attributable to managing wounds in 2012/2013 using real world evidence obtained from The Health Improvement Network (THIN) database (a nationally representative database of clinical practice among $>11$ million patients registered with general practitioners in the UK).

- The estimates were derived following a systematic analysis of patients' characteristics, wound-related health outcomes and all community-based and secondary care resource use contained in the patients' electronic records.

- Computerised information in the THIN database is collected by general practitioners (GPS) for clinical care purposes and not for research. Additionally, prescriptions issued by GPs and practice nurses are recorded in the database, but it does not specify whether the prescriptions were dispensed or patient compliance with the product.

- The analysis does not consider the potential impact of those wounds that remained unhealed beyond the study period. Nor does it consider the potential impact of managing patients with wounds being cared for in nursing homes. The THIN database may have under-recorded use of some healthcare resources outside the GP's surgery. However, the impact of this was addressed in sensitivity analyses.

infants to the elderly. The patient population with wounds is managed across the spectrum of different healthcare disciplines that includes general practice, specialist physicians, surgeons, nurses and allied healthcare practitioners, such as podiatrists. ${ }^{1-3}$

Wound care should be viewed as a specialised segment of healthcare that requires clinicians with specialist training to diagnose and manage appropriately. ${ }^{4}$ However, the evidence suggests this is not the case. ${ }^{1-3}$ Moreover, it has been suggested that better wound care, such as effective diagnosis and treatment and effective prevention of wound 
complications would help minimise treatment costs. Studies have highlighted that there is potential for better patient management and wound care product selection that would improve outcomes and reduce costs. ${ }^{1-3} 67$

There have been previous attempts to estimate the costs and burden associated with wound management in the UK. However, they were based on published literature or broad estimates derived from incidence or prevalence rates and extrapolations from relatively small-scale studies. The objectives of the present study were to estimate: the annual prevalence of wounds and annual number of wounds that are treated by the UK's publiclyfunded National Health Service (NHS); the annual amount of NHS resource use that is utilised on wound management; and the amount the NHS spends on managing wounds in $2012 / 2013$. The remit of this study was limited to open external acute and chronic cutaneous wounds. Surgical wounds healing within 4 weeks were excluded as costs were considered to be related to the surgery and not to ongoing wound complications.

\section{METHODS}

\section{Study design}

This was a retrospective cohort analysis of the records of patients in The Health Improvement Network (THIN) database.

\section{The THIN database}

The THIN database (Cegedim, London, UK) contains computerised information on $>11$ million anonymised patients entered by general practitioners (GPs) from 562 practices across the UK. General practices across the UK using Vision Practice Management Software are invited to participate in the database and are self-selecting. The patient data within THIN have been shown to be representative of the UK population in terms of demographics and disease distribution. ${ }^{8}$ Read codes are a coded thesaurus of clinical terms that have been in use in the NHS since $1985,{ }^{9}$ and have been used to code specific diagnoses in the THIN database. A drug dictionary based on data from the Multilex classification has been used to code drugs in the database. Successive updates of patients' records to the database include any subsequent changes made by GPs.

The computerised information in the THIN database includes patients' demographics, details from GP consultations, specialist referrals, nurse and other clinician visits, hospital admissions, diagnostic and therapeutic procedures, laboratory tests and prescriptions issued in primary care that are directly generated by the general practice's information technology system. Hence, the information contained in the THIN database reflects real clinical practice, as it is based on actual patient records. Moreover, GPs are the gatekeepers to healthcare in the UK, and patients' entire medical history should be stored in their primary care record.

\section{Study population}

The study population comprised the anonymised case records of a randomly selected cohort of 1000 patients from the THIN database who had a wound between 1 May 2012 and 30 April 2013 (cases) and a randomly selected cohort of 1000 control patients (controls) from the database, who were matched with the cases according to age, gender and the patient's general practice.

Inclusion criteria for the cases were:

- Had to be aged 18 years or above.

- Had to have a Read code for a wound.

- Had to have continuous medical history in their case record from the first mention of a wound in the study year up to the time the data were extracted from the database, unless they died, in order to exclude patients who had moved or changed their general practice.

Exclusion criteria for the cases were:

- Patients with a surgical wound if they healed within 4 weeks of the surgical procedure (since any resource use incurred will be due to the surgical procedure and not the wound).

- Patients with a dermatological tumour.

1000 control patients were matched with 1000 cases according to the following criteria:

- Age.

- Gender.

- Being managed at the same general practice.

- No history of a wound in their medical record at anytime.

- Had continuous medical history in their case record from the matched start date up to the time the data were extracted from the database unless they died.

The authors obtained the complete medical records of the 2000 patients in the data set, which enabled analysis of data within and outside of the study period.

\section{Ethics approval}

Ethics approval to use the complete patients' records from the THIN database for this study was obtained from Cegedim's Research Ethics Committee that appraises studies using the THIN database (Reference number 13-061).

\section{Study variables and statistical analyses}

Information was systematically extracted from the patients' records over the study period according to the protocol approved by the ethics committee. Wound type was documented in the patients' records and the authors categorised them as being either acute (ie, abscess, burn, open wound, unhealed surgical wound, trauma) or chronic (ie, diabetic foot ulcer, arterial leg ulcer, mixed leg ulcer, venous leg ulcer, pressure ulcer).

Patients' characteristics, comorbidities (defined as a non-acute condition that patients were suffering from in the year before the start of their wound and not necessarily the year before the start of the study), wound-related health outcomes and all community- 
based and secondary care resource use were extracted from the electronic records. All the data were quantified for cases and controls and stratified according to wound type. Differences between the groups were tested for statistical significance using either a Mann-Whitney U-test or a $\chi^{2}$ test.

Logistic regression was used to investigate relationships between baseline variables and clinical outcomes. Multiple linear regression was also used to assess the impact of patients' baseline variables on resource use and clinical outcomes. All statistical analyses were performed using IBM SPSS Statistics (V.22.0; IBM Corporation).

\section{Health economic modeling}

Using the THIN data set, a computer-based model was constructed depicting the treatment pathways and associated management of the 1000 patients with a wound and the 1000 matched patients who had never had a wound. The model spans the 12 months period from 1 May 2012 to 30 April 2013.

Unit costs at $2013 / 2014$ prices $^{10-12}$ were applied to the resource use in the model to estimate the total NHS cost of patient management from the time a patient entered the data set (ie, from 1 May 2012 or the start time of their wound if it occurred later, and the equivalent date in the matched control) up to the time their wound healed or the end of the study period, whichever came first. Differences between cases and controls were considered to be attributable to wound care and associated comorbidities.

\section{Sensitivity analyses}

Deterministic sensitivity analyses were performed on all of the model's inputs to identify how the cost of wound management and associated comorbidities would change by varying the different parameters in the model.

Two methods were used to adjust for the cost of managing patients' comorbidities.

1. The first involved generating an incremental cost among control patients between those who had no comorbidities and those who had one, two, three, four or five or more comorbidities. These incremental costs were then applied to both groups so that all the patients were modelled to have the maximum number of comorbidities. The resulting cost difference between the two groups was considered to be solely due to the wounds.

2. The second method involved the removal of a casecontrol match from the analysis if they did not have the same number of comorbidities. The resulting cost difference between the two groups was considered to be solely due to the wounds.

\section{RESULTS}

\section{Patients' characteristics}

Patients' mean age in the cases and controls was 69.0 and 67.3 years, respectively, and $55 \%$ of patients in both groups were female. Mean blood pressure was 133/76 in the cases and $132 / 77$ in the controls. Additionally, patients' body mass index was a mean $29.0 \mathrm{~kg} / \mathrm{m}^{2}$ and $26.1 \mathrm{~kg} / \mathrm{m}^{2}$ in the cases and controls respectively. $18 \%$ of the cases were smokers, $39 \%$ were non-smokers and $40 \%$ were ex-smokers. In the controls, $17 \%$ were smokers, $47 \%$ were non-smokers and $32 \%$ were ex-smokers. Seventy six per cent of cases presented with a new wound in the study year (patients' records predated the onset of the study period, enabling both pre-existing and new wounds to be identified. A similar process allowed wound healing to be characterised). There was no evidence that patients in the data set had more than one wound. However, $72 \%$ of patients had a wound a mean 4.9 years prior to the one being evaluated in the study period.

Significantly more patients with a wound $(94 \%)$ than control patients $(77 \%)$ had at least one comorbidity in the year before the start of their wound. Moreover, the mean number of comorbid conditions in the cases was 3.9 per patient compared to 2.1 per patient in the controls. The percentage of patients with different comorbidities in the year before the start of their wound (and not necessarily the year before the start of the study) is summarised in table 1.

Binary logistic regression was performed on patients' age, gender, smoking status and all comorbidities. Those variables that yielded a $p$ value $\geq 0.05$ were omitted from the analysis resulting in the following comorbidities being considered as independent risk factors for developing a wound:

- Dermatological symptoms: OR 3.26 (95\% CI 2.66 to $4.00) ; \mathrm{p}<0.001$.

- Nutritional deficiency: OR 2.30 (95\% CI 1.80 to $2.95) ; \mathrm{p}<0.001$.

- Musculoskeletal disease: OR 1.62 (95\% CI 1.29 to 2.03); $\mathrm{p}<0.001$.

Table 1 Percentage of patients with a comorbidity in the year before the start of their wound

\begin{tabular}{llll}
\hline \multicolumn{1}{c}{ Comorbidity } & \multicolumn{2}{l}{ Percentage of patients } & \\
\cline { 2 - 3 } & Cases & Controls & \\
Cardiovascular & 73 & $\mathbf{( \% )}$ & p Value \\
Dermatological & 59 & 53 & $<0.005$ \\
Endocrinological & 45 & 25 & $<0.001$ \\
Gastroenterological & 43 & 27 & $<0.01$ \\
Immunological & 3 & 0 & $<0.01$ \\
Musculoskeletal & 37 & 19 & $\mathrm{~ns}$ \\
Neurological & 23 & 13 & $<0.005$ \\
Nutritional & 34 & 13 & $\mathrm{~ns}$ \\
deficiency & & & $<0.001$ \\
Other & 14 & 7 & $\mathrm{~ns}$ \\
Psychiatric & 31 & 18 & $<0.05$ \\
Respiratory & 28 & 15 & $\mathrm{~ns}$ \\
None & 6 & 23 & $<0.001$ \\
\hline If the p value was $\geq 0.05$ it was considered not significant (ns).
\end{tabular}


- Cardiovascular disease: OR 1.35 (95\% CI 1.09 to $1.67) ; p=0.005$.

- Gastrointestinal disease: OR $1.32 \quad(95 \%$ CI 1.06 to $1.64) ; p=0.015$.

\section{Prevalence of wounds in the UK}

The base population of active patients in the THIN database in 2012/2013 was 3.9 million, from which there were an estimated 135000 patients with a wound that matched the study protocol's inclusion and exclusion criteria. When this was extrapolated to the whole UK population (63.7 million people in mid-2013), it was estimated that there were 2.2 million patients with a wound who matched the study protocol's inclusion and exclusion criteria, equivalent to $4.5 \%$ of the adult population, in the study year (table 2).

Twelve per cent of all wounds had no diagnosis and it was not possible to infer a wound type from the patients' records. Additionally, $19 \%$ of all wounds were a leg ulcer without any further characterisation (ie, venous, arterial or mixed). In total, there were $730000 \mathrm{leg}$ ulcers, which equates to $1.5 \%$ of the adult population having a leg ulcer in the study year. The number of diagnosed venous leg ulcers (278 000) indicates that 1 in 170 adults had such an ulcer in the study year. There were also an estimated 169000 diabetic foot ulcers, which equates to $5 \%$ of adult diabetic patients having a foot ulcer in the study year, of which $66 \%$ were male. There was no evidence that any of the patients in our data set had more than one wound.

\section{Clinical outcomes}

Sixty one per cent of all wounds healed in the study year; $79 \%$ of acute wounds healed and $43 \%$ of chronic

Table 2 Annual number and prevalence of different wound types in the UK

\begin{tabular}{|c|c|c|}
\hline & $\begin{array}{l}\text { Annual number } \\
\text { of wounds }\end{array}$ & $\begin{array}{l}\text { Annual } \\
\text { prevalence } \\
\text { among the adult } \\
\text { UK population }\end{array}$ \\
\hline Abscess & $160000(7 \%)$ & 0.0032 \\
\hline Burn & 87000 (4\%) & 0.0018 \\
\hline Diabetic foot ulcer & 169000 (8\%) & 0.0034 \\
\hline Leg ulcer (arterial) & $9000(<1 \%)$ & 0.0002 \\
\hline Leg ulcer (mixed) & 24000 (1\%) & 0.0005 \\
\hline $\begin{array}{l}\text { Leg ulcer } \\
\text { (unspecified) }\end{array}$ & $420000(19 \%)$ & 0.0085 \\
\hline Leg ulcer (venous) & 278000 (13\%) & 0.0056 \\
\hline Open wound & 240000 (11\%) & 0.0048 \\
\hline Pressure ulcer & $153000(7 \%)$ & 0.0031 \\
\hline Surgical wound & 253000 (11\%) & 0.0051 \\
\hline Trauma & $158000(7 \%)$ & 0.0032 \\
\hline Unspecified & 271000 (12\%) & 0.0055 \\
\hline Total & 2222000 (100\%) & 0.0447 \\
\hline \multicolumn{3}{|c|}{$\begin{array}{l}\text { Among the adult population (ie, } \geq 18 \text { years of age) estimated from } \\
\text { the THIN database. } \\
\text { THIN, The Health Improvement Network. } \\
\text { Percentage of total number of wounds is in parentheses. }\end{array}$} \\
\hline
\end{tabular}

wounds. Binary logistic regression suggests that nutritional deficiency (OR $0.53 \quad(95 \%$ CI 0.41 to 0.70$)$; $\mathrm{p}<0.001)$ and diabetes (OR $0.65(95 \%$ CI 0.50 to 0.85$)$; $\mathrm{p}<0.001)$ were independent risk factors for non-healing during the study period. Additionally, $4 \%$ of patients with a wound and $1 \%$ of control patients died in the study year.

\section{Healthcare resource use associated with patient management}

Patients in both groups were predominantly managed in the community by GPs and nurses. Table 3 summarises the percentage of patients in each group who utilised different resources during the study year.

Table 4 shows the annual number of resources associated with managing 2.2 million patients with a wound and 2.2 million control patients. The differences were considered to be the incremental amounts of resource use attributable to managing 2.2 million wounds and associated comorbidities and included 18.6 million practice nurse visits, 10.9 million community nurse visits, 7.7 million GP visits, 3.4 million hospital outpatient visits, 97.1 million drug prescriptions, 262.2 million dressings, 73.4 million bandages and 9.0 million compression bandages. Assessment of peripheral perfusion is a recognised requirement for leg ulcer and diabetic foot management, yet only $16 \%$ of all cases with a leg or foot ulcer had a Doppler ankle brachial pressure index recorded in their records.

The total annual NHS cost of managing 2.2 million patients with a wound was estimated to be $£ 6.0$ billion (table 5). The corresponding cost of managing the matched controls was $£ 0.7$ billion. Hence, the total annual NHS cost of managing 2.2 million wounds and associated comorbidities was estimated to be $£ 5.3$ billion. Of this, £2.1 billion and £3.2 billion was associated with managing those wounds that healed and remained unhealed respectively.

Sixty six per cent of the total annual NHS cost was incurred in the community and the remainder in secondary care. However, the distribution of costs varied according to wound type, with $48 \%$ and $78 \%$ of the total annual NHS cost of managing acute and chronic wounds, respectively, being incurred in the community and the remainder in secondary care.

\section{Sensitivity analyses}

The estimated amounts of individual resource use were reduced and increased by $25 \%$. However, this only affected the total annual NHS cost of managing 2.2 million wounds and associated comorbidities by $6 \%$ or less.

The cost of an initial surgical procedure may not be relevant to the cost incurred in managing an unhealed surgical wound. Therefore, a sensitivity analysis was performed to exclude this cost from the cost of managing patients with an unhealed surgical wound. The analysis found that when the cost of surgery was excluded from the cost of managing patients with an unhealed surgical 
Table 3 Percentage of patients who utilised resources in the study year

\begin{tabular}{|c|c|c|c|}
\hline \multirow[b]{2}{*}{ Resource } & \multicolumn{2}{|c|}{ Percentage of patients } & \multirow[b]{2}{*}{ p Value } \\
\hline & Cases (\%) & Controls (\%) & \\
\hline GP visits & 86 & 47 & $<0.001$ \\
\hline Practice nurse visits & 72 & 29 & $<0.001$ \\
\hline Community nurse visits & 75 & 2 & $<0.001$ \\
\hline Specialist nurse visits & 2 & $<1$ & ns \\
\hline Allied healthcare visits & 14 & 3 & 0.005 \\
\hline Hospital outpatient visits & 53 & 18 & $<0.001$ \\
\hline Hospital admissions and day cases & 29 & 6 & $<0.001$ \\
\hline Ambulance services & $<1$ & $<1$ & ns \\
\hline Accident and emergency attendances & $<1$ & $<1$ & ns \\
\hline Diagnostic tests & 80 & 45 & $<0.001$ \\
\hline Non-wound care devices & 36 & 5 & $<0.001$ \\
\hline Wound care products & 100 & 0 & $<0.001$ \\
\hline Prescriptions for individual drugs & 98 & 72 & $<0.001$ \\
\hline
\end{tabular}

wound, the total annual NHS cost of managing 2.2 million wounds and associated comorbidities was reduced from $£ 5.3$ billion to $£ 4.8$ billion.

When the NHS cost of managing patients was adjusted for their comorbidities (see description of method 1 and 2 sections under Sensitivity analyses), the total annual NHS cost of managing 2.2 million wounds was reduced from $£ 5.3$ billion to $£ 5.1$ billion when method 1 was used and to $£ 4.5$ billion when method 2 was used. Hence, the total annual NHS cost of managing the comorbidities among 2.2 million patients with a wound was estimated to be between $£ 250$ and $£ 788$ million. After adjusting for comorbidities, the total annual NHS cost of managing healed wounds and unhealed wounds was estimated to be $£ 2.0$ billion and $£ 3.0$ billion, respectively.

\section{DISCUSSION}

This study estimated the health outcomes, resource implications and associated costs attributable to managing wounds in 2012/2013. After adjustment for comorbidities, the annual NHS cost of managing wounds was estimated to be $£ 4.5-5.1$ billion, two-thirds of which is incurred in the community and the rest in secondary care. This is comparable to the annual NHS cost of managing obesity which was estimated at $£ 5.0$ billion in 2013 . $^{13}$

For patients to be included in the data set, they had to have had a wound in the study year or be matched to these patients on the basis of their age, sex, the same general practice, and no evidence of a wound in their medical history. Patients in both groups were managed primarily by clinicians in the community. Only half the patients with a wound saw a hospital physician although the consultation may not have been wound-related. However, the evidence shows that wound management is predominantly a nurse-led discipline. Approximately $30 \%$ of wounds lacked a differential diagnosis. This may be indicative of the practical difficulties experienced by non-specialist healthcare professionals in the community

Table 4 Annual amount of NHS resource use attributable to managing 2.2 million patients with a wound and 2.2 million matched controls

\begin{tabular}{|c|c|c|c|c|}
\hline \multirow[b]{2}{*}{ Resource } & \multicolumn{3}{|c|}{ Annual number } & \multirow[b]{2}{*}{ p Value } \\
\hline & Cases & Controls & Difference & \\
\hline GP visits & 10816655 & 3124120 & 7692535 & $<0.001$ \\
\hline Practice nurse visits & 19744618 & 1184322 & 18560296 & $<0.001$ \\
\hline Community nurse visits & 10932199 & 75548 & 10856651 & $<0.001$ \\
\hline Specialist nurse visits & 51106 & 4444 & 46662 & $<0.001$ \\
\hline Allied healthcare visits & 537722 & 77770 & 459952 & $<0.001$ \\
\hline Hospital outpatient visits & 4277334 & 828803 & 3448531 & $<0.001$ \\
\hline Hospital admissions and day cases & 1142104 & 173315 & 968788 & $<0.001$ \\
\hline Ambulance services & 11110 & 2222 & 8888 & ns \\
\hline Accident and emergency attendances & 11110 & 11110 & - & ns \\
\hline Diagnostic tests & 60284855 & 24068613 & 36216242 & $<0.001$ \\
\hline Devices & 320938916 & 48206108 & 272732808 & $<0.001$ \\
\hline Wound care products & 354954275 & 0 & 354954275 & $<0.001$ \\
\hline Prescriptions for individual drugs & 135859234 & 38769310 & 97089924 & $<0.001$ \\
\hline
\end{tabular}


Table 5 Annual cost of NHS resource use attributable to managing 2.2 million patients with a wound and 2.2 million matched controls

\begin{tabular}{llll}
\hline & Annual cost & & \\
\cline { 2 - 4 } Resource & Cases & Controls & Difference \\
\hline GP visits & $£ 514993223$ & $£ 145951520$ & $£ 369041702$ \\
Practice nurse visits & $£ 256760021$ & $£ 15396180$ & $£ 241363841$ \\
Community nurse visits & $£ 682382518$ & $£ 3026353$ & $£ 679356166$ \\
Specialist nurse visits & $£ 3650732$ & $£ 322189$ & $£ 3328543$ \\
Allied healthcare visits & $£ 34451980$ & $£ 4859496$ & $£ 29592485$ \\
Hospital outpatient visits & $£ 515002111$ & $£ 99947406$ & $£ 415054705$ \\
Hospital admissions and day cases & $£ 1334299309$ & $£ 135277073$ & $£ 1199022237$ \\
Ambulance services & $£ 2555290$ & $£ 511058$ & $£ 2044232$ \\
Accident and emergency attendances & $£ 666597$ & $£ 666597$ & $£ 0$ \\
Diagnostic tests & $£ 282646224$ & $£ 113238466$ & $£ 169407758$ \\
Devices & $£ 282261975$ & $£ 17525292$ & $£ 264736682$ \\
Wound care products & $£ 742703819$ & $£ 0$ & $£ 742703819$ \\
Prescriptions for individual drugs & $£ 1390246214$ & $£ 188175050$ & $£ 1202071164$ \\
TOTAL & $£ 6042620014$ & $£ 724896679$ & $£ 5317723335$ \\
\hline GP, general practitioner; NHS, National Health Service. & & &
\end{tabular}

with establishing a working diagnosis. Moreover, it appears that only $16 \%$ of patients with a leg or foot ulcer had a Doppler. However, national guidance in the UK for both leg ulcer management and the management of diabetic foot ulceration requires arterial assessment by Doppler ultrasound measurement of the ankle-brachial pressure index. ${ }^{14} 15$ Hence, these findings suggest (1) the need to refer patients to a specialist for investigation and a differential diagnosis and a shared management plan to be implemented in the community and (2) training of non-specialist clinicians in the fundamentals of wound management. These two measures should help overcome some of the problems encountered in clinical practice and achieve better health outcomes, thereby reducing the high levels of resource use and corresponding costs, and improving compliance with appropriate National Institute for Health and Care Excellence (NICE) and best practice guidelines. The study highlighted that despite being a nurse-led discipline, there is a lack of involvement of tissue viability nurses and other specialist nurses in the management of patients with wounds. Recent articles ${ }^{16}{ }^{17}$ highlight the lack of a clear definition, and therefore confusion regarding the role of these nurses.

The wound patients had significantly more comorbidities than their matched controls. However, it was surprising to find such a high level of illness among the controls. For example, 53\% had cardiovascular disease. Moreover, $34 \%$ of patients with a wound and $13 \%$ of controls had nutritional deficiency that warranted supplementation with clinical nutritional formulae. The THIN data set in this study covers the period 2012/2013 and comprises patients with a wide age range (19-98 years), so this level of illness may be a proxy for the health of a proportion of the general population at that time. Furthermore, the presence of nutritional deficiency was an independent risk factor for wound non-healing. Hence, community-prevention of patients developing nutritional deficiency should help improve healing rates following the onset of a wound. Moreover, if patients' wounds and their comorbidities were treated more holistically with appropriate involvement of allied healthcare professionals, rather than just focusing on the wounds, better health outcomes might be achieved at lower cost.

Other studies have assessed the prevalence and costs of wound care. A district-wide survey in the Bradford and Airedale area of the UK estimated the annual NHS cost of wound care to be $£ 2.0$ million/100 000 population, ${ }^{18}$ which equates to $£ 1.3$ billion for the UK as a whole. In another study, the NHS cost of caring for patients with a chronic wound was estimated to be £2.33.1 billion per year at 2005/2006 prices. ${ }^{19}$ Previous prevalence studies suggest there may be up to 190000 individuals with a venous leg ulcer in the UK at any time. ${ }^{19}$ However, this is likely to be an underestimate as our analysis estimated that the NHS manages 278000 venous leg ulcers per annum plus an additional 420000 unspecified leg ulcers (ie, those lacking a true working diagnosis), some of which will undoubtedly be venous in origin. The prevalence of pressure ulcers has been previously estimated to be 0.103 in $2005 / 2006^{20}$ and another study estimated the incidence to be 400000 new pressure ulcers per annum in the UK. ${ }^{19}$ These figures are substantially higher than our estimate of the NHS managing 153000 pressure ulcers per annum. The difference may be due to people residing in nursing homes not having been included in our study. It may also be due to under-recording of pressure ulcers in patients' records and/or an over-estimation in the previous studies, and/or a lower prevalence in 2012/2013 than in $2005 / 2006$. The present study also estimated that $5 \%$ of patients with diabetes have a foot ulcer which is consistent with other estimates. ${ }^{21}$ 
The advantage of using the THIN database is that the patient pathways and associated resource use are based on real world evidence derived from clinical practice. However, health outcomes and resource use, while collected prospectively, were analysed retrospectively. Moreover, there was no intention to match patients for comorbidities, since differences in comorbidities between the groups were an intended outcome of the study. Sensitivity analyses estimated that the cost of managing the comorbidities ranged between $£ 250$ and $£ 788$ million. Nevertheless, the possibility of resource use associated with managing a comorbidity being conflated with that of wound management cannot be excluded. Hence, confounding factors may exist. While the study results are compelling, the analyses were based on clinicians' entries into their patients' records and inevitably subject to a certain amount of imprecision and lack of detail. Moreover, the computerised information in the THIN database is collected by GPs for clinical care purposes and not for research. Prescriptions issued by GPs and practice nurses are recorded in the database, but it does not specify whether the prescriptions were dispensed or patient compliance with the product. Despite these limitations, it is the authors' opinion that the THIN database affords one of the best sources of real world evidence for clinical practice in the UK.

The analysis does not consider the potential impact of those wounds that remained unhealed beyond the study period, nor does it consider the potential impact of managing patients with wounds being cared for in nursing/residential homes. The THIN database may have under-recorded use of some healthcare resources outside the GP's surgery if not documented in the GP records, such as home visits made by clinicians, hospital outpatient visits, hospital admissions and accident and emergency attendances. The impact of this was addressed in sensitivity analyses. The analysis excludes hospital-based prescribing, but this should have minimal impact on the results since most prescribing is undertaken by GPs and nurses in the community.

The analysis only considered the annual cost of NHS resource use for the 'average patient,' and no attempt was made to stratify resource use and costs according to gender, comorbidities, wound size, wound severity and other disease-related factors. Also excluded were the costs incurred by patients and indirect costs incurred by society as a result of patients taking time off work. However, patients' mean age was $>65$ years, so it is unlikely that many were in employment.

Notwithstanding the study's limitations, the real world evidence in our study demonstrates that wounds impose a substantial health economic burden on the UK's NHS, comparable to that of managing obesity. Clinical and economic benefits could accrue from improved systems of care and an increased awareness of the impact that wounds impose on patients and the NHS.
Contributors JFG designed the study, managed the analyses, performed some analyses, checked all the other analyses, and wrote the manuscript. NA and TM conducted much of the analyses. IU and AG conducted some of the analyses. DW scrutinised the analyses and edited the manuscript. KV and PV scrutinised the analyses, suggested further analyses, helped interpret some of the findings, and edited the manuscript. JFG is the guarantor of this work and, as such, had full access to all the data in the study and takes responsibility for the integrity of the data and the accuracy of the data analysis. The analyses have also been reviewed by the following strategic partners of the NIHR Wound Prevention and Treatment Healthcare Technology Co-operative (NIHR WoundTec HTC) (http://www.woundtec.htc.nihr.ac.uk): Professor Andrea Nelson, Head of School of Healthcare, University of Leeds, Leeds; Professor Jane Nixon, Deputy Director, Leeds Institute of Clinical Trials Research, Leeds; Professor Dan L Bader, Faculty of Health Sciences, University of Southampton, Southampton; Dr Patricia Grocott, Reader in Palliative Wound Care Technology Transfer, King's College, London; Hussein Dharma, Project Manager, NIHR WoundTec Healthcare Technology Co-operative, Bradford Institute for Health Research, Bradford.

Funding This study was commisioned and funded by the NIHR Wound Prevention and Treatment Healthcare Technology Co-operative (NIHR WoundTec HTC), Bradford Institute For Health Research, Bradford, West Yorkshire, UK. following an open tendering process. Additional funding was provided by 3M Health Care Limited, Loughborough, Leicestershire, UK; Activa Healthcare Limited, Burton On Trent, Staffordshire, UK; Brightwake Limited, Kirkby In Ashfield, Nottinghamshire, UK; KCI Medical Limited, Crawley, West Sussex, UK; Longhand Data, Welburn, North Yorkshire, UK; Medira Limited, Cambridge, Cambridgeshire, UK; Molnlycke Health Care Limited, Dunstable, Bedfordshire, UK; Park House Healthcare Limited, Elland, West Yorkshire, UK; Perfectus Biomed Limited, Daresbury, Warrington, UK; Pulsecare Medical LLC, North Andover, Massachusets, USA; Smith \& Nephew Medical Limited, Hull, East Riding Of Yorkshire, UK; Sozo Woundcare Limited, Harrogate, North Yorkshire, UK; Systagenix Wound Management Limited, Gatwick Airport, West Sussex, UK; Trio Healthcare, Great Missenden, Buckinghamshire, UK; Urgo Limited, Loughborough, Leicestershire, UK; Willingsford Limited, Southampton, Hampshire, UK.

\section{Competing interests None declared.}

Disclaimer The study's sponsors had no involvement in the study design, the collection, analysis and interpretation of the data, the writing of this manuscript and the decision to submit this article for publication. The views expressed in this article are those of the authors and not necessarily those of the NHS, the NIHR, the Department of Health or any of the other sponsors.

Ethics approval Cegedim's Research Ethics Committee that appraises studies using the THIN database (Reference number 13-061).

Provenance and peer review Not commissioned; externally peer reviewed.

Data sharing statement No additional data are available.

Open Access This is an Open Access article distributed in accordance with the Creative Commons Attribution Non Commercial (CC BY-NC 4.0) license, which permits others to distribute, remix, adapt, build upon this work noncommercially, and license their derivative works on different terms, provided the original work is properly cited and the use is non-commercial. See: http:// creativecommons.org/licenses/by-nc/4.0/

\section{REFERENCES}

1. Guest JF, Taylor RR, Vowden K, et al. Relative cost-effectiveness of a skin protectant in managing venous leg ulcers in the UK. $J$ Wound Care 2012;21:389-98.

2. Panca M, Cutting K, Guest JF. Clinical and cost-effectiveness of absorbent dressings in the treatment of highly exuding VLUs. $J$ Wound Care 2013;22:109-18.

3. Guest JF, Gerrish A, Ayoub N, et al. Clinical outcomes and cost-effectiveness of three alternative compression systems used in the management of venous leg ulcers. $J$ Wound Care 2015;24:300-8.

4. Harding K. 'Woundology'- an emerging clinical specialty. Int Wound J 2008;5:597. 
5. Kapp S, Santamaria N. Chronic wounds should be one of Australia's National Health Priority Areas. Australian Health Review 2015;39: 600-2.

6. Guest JF, Charles H, Cutting K. Is it time to re-appraise the role of compression in non-healing venous leg ulcers? J Wound Care 2013;22:453-60.

7. National Institute for Health and Care Excellence. NICE advice [KTT14] January 2015. https://www.nice.org.uk/advice/ ktt14/ chapter/Evidence-context (accessed 14 May 2015).

8. Blak BT, Thompson M, Dattani H, et al. Generalisability of The Health Improvement Network (THIN) database: demographics, chronic disease prevalence and mortality rates. Inform Prim Care 2011;19:251-5.

9. Read Codes. Department for Health. 2010. http://systems.hscic.gov. uk/data/uktc/readcodes (accessed 12 May 2015).

10. Department of Health. NHS reference costs 2013/14 [article online] https://www.gov.uk/government/publications/nhs-reference-costs2013-to-2014 (accessed 25 Apr 2015).

11. Curtis L. Unit Costs of Health and Social Care 2014. Canterbury: University of Kent. Personal Social Services Research Unit, 2014. http://www.pssru.ac.uk/ project-pages/unit-costs/2014 (accessed 25 Apr 2015).

12. Drug Tariff, 2014. https://www.drugtariff.co.uk (accessed 25 Apr 2015)

13. Royal College of Physicians 2013. Action on obesity: Comprehensive care for all Report of a working party January 2013.
http://www.evidence.nhs.uk/search?q=obesity\%20AND\%20costs\% 20nhs (accessed 26 Apr 2015).

14. SIGN Guideline 120: Management of chronic venous leg ulcers. Scottish Intercollegiate Guidelines Network 2010. http://www.sign.ac. uk/pdf/sign120.pdf

15. Diabetic foot problems: prevention and management. NICE guidelines [NG19]. National Institute for Health and Care Excellence (NICE), 2015

16. Ousey K, Atkin L, Milne J, et al. The changing role of the tissue viability nurse: an exploration of this multifaceted role. Wounds UK 2014;10:54-61.

17. Ousey $\mathrm{K}$, Milne J, Atkin L, et al. Exploring the role of the Tissue Viability Nurse. Wounds UK (EWMA Special) 2015: 36-45.

18. Vowden $\mathrm{K}$, Vowden $\mathrm{P}$, Posnett J. The resource cost of wound care in the Bradford and Airedale primary care trust in the UK. $J$ Wound Care 2009;18:93-4.

19. Posnett J, Franks P. The burden of chronic wounds in the UK. Nurs Times 2008;104:44-5.

20. Philips L, Busby J. Pressure Ulcer Epidemiology in the UK: 20052006. Poster Presentation at Wounds UK, 2006.

21. Diabetes in the UK 2012: Key statistics on diabetes. http://www. diabetes.org.uk/About us/What-we-say/Statistics/Diabetes-in-theUK-2012 (accessed 12 May 2015). 